\title{
KLOE/KLOE-2 results and perspectives on dark force search
}

\section{Elena Pérez del Río* for the KLOE-2 Collaboration}

LNF-INFN Via E. Fermi 40, 00044 Frascati, Italy E-mail: eperez@lnf. infn. it

\begin{abstract}
During the last years several Dark Sector Models have been proposed in order to address striking astrophysical observations which fail standard interpretations.

In the minimal case a new vector particle, the so called dark photon or U-boson, is introduced, with small coupling with Standard Model particles. Also, the existence of a dark Higgs boson $\mathrm{h}$ ' is postulated, in analogy with the Standard Model, to give mass to the U-boson through the Spontaneous Symmetry Breaking mechanism.

The experiment KLOE, working on the DA $\Phi N E$ e+e- collider in Frascati, searched for the existence of the U-boson in a quite complete way, investigating several different processes and final states. Tight limits on the model parameters have been set at 90\%CL. Further improvements are expected in terms of sensitivity and discovery potential with the new KLOE-2 detector working on the improved DAFNE e+e- collider.
\end{abstract}

The 39th International Conference on High Energy Physics (ICHEP2018)

4-11 July, 2018

Seoul, Korea

${ }^{*}$ Speaker. 


\section{Introduction}

The Standard Model (SM) does not provide a definitive model of all elementary particles. In particular, some astrophysical observations $[1,2,3,4,5,6,7,8]$ and the muon magnetic discrepancy $a_{\mu}$ are examples of possible physics beyond the SM. Extensions of the SM [9, 10, 11, 12, 13] claim to explain the afore-mentioned anomalies by means of dark matter models, with a Weakly Interacting Massive Particle (WIMP) belonging to a secluded gauge sector. The new gauge interaction would be mediated by a new vector gauge boson, the $U$ boson or dark photon, which could interact with the photon via a kinetic-mixing term $\varepsilon^{2}$. In the following, some of the $U$ boson searches, carried out with the KLOE detector, are described.

\section{2. $U$-boson search in $e^{+} e^{-} \rightarrow U \gamma$ with $U \rightarrow e^{+} e^{-}$}

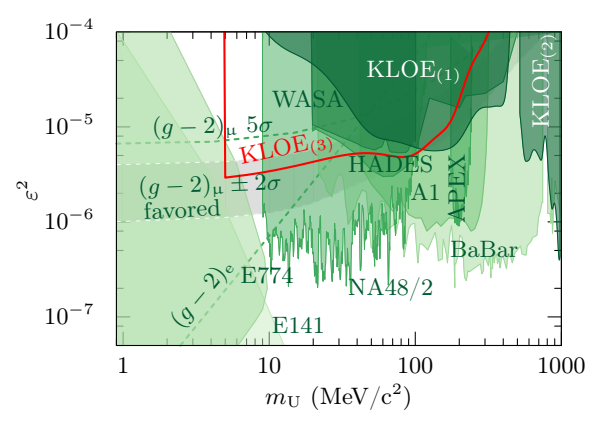

Figure 1: Exclusion limits on the kinetic-mixing parameter, $\varepsilon^{2}$, from KLOE: $\mathrm{KLOE}_{1}, \mathrm{KLOE}_{2}$ and $\mathrm{KLOE}_{3}$ (in red) correspond to the combined limits from the analysis of $\phi \rightarrow \eta e^{+} e^{-}, e^{+} e^{-} \rightarrow \mu^{+} \mu^{-} \gamma$ and $e^{+} e^{-} \rightarrow$ $e^{+} e^{-} \gamma$, respectively. The results are compared with the limits from E141, E774 [21], MAMI/A1 [22], APEX [23], WASA [24], HADES [25], NA48/2 [26] and BaBar [27]. The grey band indicates the parameter space favored by the $\left(\mathrm{g}_{\mu}-2\right)$ discrepancy.

The study of the reaction $e^{+} e^{-} \rightarrow U \gamma, U \rightarrow e^{+} e^{-}$has the characteristic that allows to investigate the low mass region close to the di-electron mass threshold [18]. The signal of the $U$ boson would be then expected as a resonant peak in the di-electron invariant mass. Since no signal was observed, the upper limit of the kinetic-mixing parameter as a function of $m_{U}$ was evaluated with the CLs technique setting a limit on the $U$-boson signal at $90 \%$ confidence level Fig. 1 . The integrated luminosity corresponds to $L_{\text {integrated }}=1.54 \mathrm{fb}^{-1}$ from the 2004-2005 data campaign.

\section{3. $U$-boson search in $e^{+} e^{-} \rightarrow U \gamma$ with $U \rightarrow \pi^{+} \pi^{-}$}

The leptonic channels investigated by KLOE loose sensitivity in the $\rho-\omega$ region due to the dominant branching fraction into hadrons. The effective coupling of the $U$ boson is predicted to be given by the product of the virtual-photon coupling and the kinetic-mixing parameter $\varepsilon^{2} e F_{\pi}\left(q^{2}\right)$ [17]. For this search, a total integrated luminosity of $1.93 \mathrm{fb}^{-1}$ was analyzed [28]. No signal was observed and a limit at the $90 \% \mathrm{CL}$ was set on the coupling factor $\varepsilon^{2}$ in the energy range between 527 and $987 \mathrm{MeV}$ with a larger sensitivity than previous limits in the $\rho-\omega$ region and above, see Fig. 2. 



Figure 2: Left: $90 \% \mathrm{CL}$ upper limit exclusion plot for $\varepsilon^{2}$ as a function of the $U$-boson mass $\left(K L O E_{(4)}\right)$. Right: $90 \% \mathrm{CL}$ exclusion plot for $\varepsilon^{2}$ as a function of the $U$-boson mass for the $e^{+} e^{-} \rightarrow U \gamma$ process. The $U \rightarrow \mu^{+} \mu^{-}$limit (dashed line), the $U \rightarrow \pi^{+} \pi^{-}$[28] constraint (solid line), and the $U \rightarrow \mu^{+} \mu^{-}, \pi^{+} \pi^{-}$ combination (blue area) at full KLOE statistics are presented in comparison with the competitive limits by BaBar [20], NA48/2 [26] and LHCb experiments [30]. The limits are shown together with previous KLOE results as well as other experiments at the moment of publication.

\section{Combined limit in the production of $U$ decaying into $\mu^{+} \mu^{-}$and $\pi^{+} \pi^{-}$}

A previous search for the $U$ boson in the decay into $\mu^{+} \mu^{-}$[31] has been extended by using the full KLOE statistics at $L_{i n t}=1.93 \mathrm{fb}^{-1}$, updating the analysis with a new estimate of the background, analogous to the one used for the $U \rightarrow \pi^{+} \pi^{-}$search. This new search confirms the non existence of $U$-boson signal in the di-muon invariant mass spectrum. To increase the sensitivity in the region of the $\rho-\omega$ interference, both results on the $90 \%$ upper limit for $\mu \mu$ and $\pi \pi$ have been combined, giving the up-to-date most stringent upper limit for the mixing parameter $\varepsilon^{2}$ in the $U$-boson mass region 519-987 MeV. The limit is shown in Fig. 2, together with the other most competitive limits in the region.

\section{Conclusions}

The KLOE collaboration has extensively contributed to the $U$-boson searches. Up to now, no evidence for a $U$ boson was found and limits at the $90 \%$ confidence level were set on the kineticmixing parameter $\varepsilon^{2}$ in the mass range $5 \mathrm{MeV}<m_{U}<987 \mathrm{MeV}$. In the meantime, a new data campaign has been finalized with the KLOE-2 setup, which has collected more than $5 \mathrm{fb}^{-1}$ in the past three years. The new setup and the enlarged statistics could further improve the current limits on the dark coupling constant by at least a factor of two.

\section{Acknowledgements}

We warmly thank our former KLOE colleagues for the access to the data collected during the KLOE data-taking campaign. We thank the DAФNE team for their efforts in maintaining low background running conditions and their collaboration during all data taking. We want to thank our technical staff: G.F. Fortugno and F. Sborzacchi for their dedication in ensuring efficient operation of the KLOE computing facilities; M. Anelli for his continuous attention to the gas 
system and detector safety; A. Balla, M. Gatta, G. Corradi and G. Papalino for electronics maintenance; C. Piscitelli for his help during major maintenance periods. This work was supported in part by the Polish National Science Centre through the Grants No.

2013/11/B/ST2/04245, 2014/14/E/ST2/00262, 2014/12/S/ST2/00459, 2016/21/N/ST2/01727, 2016/23/N/ST2/01293, 2017/26/M/ST2/00697.

\section{References}

[1] P. Jean et al., Astrophys. 407, L55 (2003).

[2] C. E. Aalseth, et al., Phys. Rev. Lett. 107, 141301 (2011).

[3] R. Bernabei, et al., Int. J. Mod. Phys. D13, 2127 (2004).

[4] R. Bernabei, et al., Eur. Phys. J. C56, 333 (2008).

[5] J. Chang, et al. Nature 456, 362 (2008).

[6] F. Aharonian, et al., Phys. Rev. Lett. 101, 261104 (2008).

[7] F. Aharonian, et al., Astron. Astrophys. 508, 561 (2009).

[8] A. A. Abdo, et al., Phys. Rev. Lett. 102, 181101 (2009).

[9] B. Holdom, Phys. Lett. B166, 196 (1985).

[10] C. Boehm, P. Fayet, Nucl. Phys. B683, 219 (2004).

[11] P. Fayet, Phys. Rev. D75, 115017 (2007).

[12] M. Pospelov, A. Ritz, M.B. Voloshin, Phys. Lett. B662, 53 (2008).

[13] Y. Mambrini,J. Cosmol. Astropart. Phys. 1009, 022 (2010).

[14] A. Gallo, et al., DAFNE status report, Conf. Proc. C060626 (2006) 604âAŞ606.

[15] M. Adinolfi et al., Nucl. Instrum. Methods A488, 51 (2002)

[16] M. Adinolfi et al., Nucl. Instrum. Methods A482, 364 (2002)

[17] M. Reece, L.T. Wang, JHEP 07, 51 (2009).

[18] A. Anastasi, et al., Physics Letters B750, 633âĂŞ637 (2015).

[19] L. Barzé et all., Eur. Phys. J. C71, 1680 (2011).

[20] J.P. Lees et al., Phys. Rev. Lett. 108, 211801 (2012).

[21] J. D. Bjorken, et al., Phys. Rev. D 80, 075018 (2009).

[22] H. Merkel et al., Phys. Rev. Lett. [112, 221802 (2014).

[23] S. Abrahamyan et al., Phys. Rev. Lett. 107, 191804 (2011).

[24] P. Adlarson et al., Phys. Lett. B726, 187 (2013).

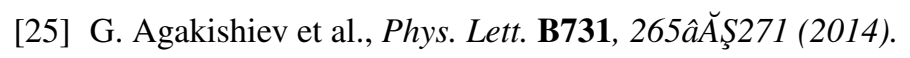



[27] J.P. Lees et al., Phys. Rev. Lett. 113, 201801 (2014).

[28] A. Anastasi et al., Phys. Lett. $\mathbf{B 7 5 7}$ (2016) 356-361

[29] G. Gounaris, J.J. Sakurai, Phys. Rev. Lett. 21 (1968) 244.

[30] S. Abrahamyan et al., Phys. Rev. Lett. 107 (2011) 191804.

[31] D. Babusci et al., Phys. Lett. B736, 459-464 (2014). 